\title{
Die soziale Kommunikation Stiefkind des kanonischen Rechts (cc. 822-832)
} von André und Cornelia Ruszkowski

Es ist das erste Mal, daß ein Codex des Kanonischen Rechtes Bestimmungen über die sozialen Kommunikationsmittel enthält ${ }^{1}$. Daher ist es nicht verwunderlich, daß man dies grundsätzlich zwar mit großer Genugtuung registriert, andererseits aber auch gewissermaßen enttäuscht ist, wenn man die betreffenden Texte an den gehegten Erwartungen mißt. Zumal wenn mehr als ein halbes Jahrhundert lang daran gearbeitet wurde, eine aktivere Teilnahme der Gläubigen an der expansiven Entwicklung der Medien zu erreichen. Diese Bemühungen, die allerdings auch erfolgreich gewesen sind, und sich zu vielfachen kirchlichen Aktivitäten und richtungsweisenden Vorgaben auf den verschiedenen Ebenen kirchlicher Strukturen ${ }^{2}$ konkretisiert haben, hätten durchaus auch im Codex von 1983 einen deutlicheren Niederschlag finden können.

Auf den folgenden Seiten möchten wir zunächst den Blick auf die Aussagen des Codex über die sozialen Kommunikationsmittel lenken und dann auf einige Gesichtspunkte hinweisen, die unberücksichtigt geblieben sind. Diese Vorgehensweise führt nun sowohl zu einigen Schlußfolgerungen hinsichtlich wünschenswerter Verbesserungen im Codex selbst, als auch dazu, die kirchliche Grundhaltung gegenüber den Medien einer notwendigen Überprüfung zu unterziehen.

Die vorliegende Ausarbeitung erhebt keinerlei Anspruch auf Wissenschaftlichkeit. Sie stellt den Standpunkt von Laien dar, die sich aktiv für das, was früher einmal „Medienapostolat" hieß, engagieren; sie sind eher durch ihre Erfahrung als durch Lektüre dazu gekommen. Zudem ist über diesen spezifischen Teil des Codex nicht allzuviel veröffentlich worden. Die einzige diesbezügliche Studie, die wir kennen, ist die von E. Baragli. Aber niemand hat deutlich genug auf deren Bedeutung für die kirchlichen Lehraussagen über die sozialen Kommunikationsmittel ${ }^{3}$ hingewiesen. Dieser Beitrag war uns eine große Hilfe bei unserer Arbeit, und wir sind dem Autor sehr dankbar, daß er ihn uns zur Verfügung gestellt hat. Das mindert jedoch nicht unsere Verantwortlichkeit für den hier vertretenen Standpunkt.

\section{Die Aussagen des Codex}

Wer nach den medienrelevanten Aussagen des Codex sucht, wird zunächst nach einem Kapitel Ausschau halten, in dem mehrere Canones unter

André Ruszkowski ist Professor emeritus des 'Institut des communications sociales'der Universität Saint-Paul, Ottawa/Kanada. Der Beitrag wurde in französischer Sprache in den „Studia Canonica“ 23 (1989), S. 471-484, Ottawa, publiziert. Prof. Ruszkowski hat ihn für CS zur Verfügung gestellt. 
einer entsprechenden Überschrift zusammengefaßt sind. Ein solches findet sich im Buch III: Verkündigungsdienst der Kirchen. Titel IV ist überschrieben: „Soziale Kommunikationsmittel. Insbesondere Bücher" (cc. 822-832). Jedoch wäre es falsch, sich darauf zu beschränken, weil man bei aufmerksamer Prüfung des Codex noch eine Reihe anderer Textstellen über die sozialen Kommunikationsmittel entdecken kann, die man nicht übersehen sollte. Wir wenden uns jedoch zunächst dem Titel IV des III. Buches zu und werden ihn dann auch im Licht der übrigen medienrelevanten Textstellen betrachten.

\subsection{Kommunikation oder Zensur?}

Was zunächst einen Experten wie Baragli in Erstaunen versetzt, wenn er die Überschrift des Titels IV im III. Buch liest ist, daß man dem Begriff „Soziale Kommunikationsmittel“ noch hinzugefügt hat: „Insbesondere Bücher“. Nach der in Fachkreisen vorherrschenden Ansicht ist 'Masse' Bestandteil des Begriffes der sozialen Kommunikationsmittel. Allerdings richtet sich das Medium Buch nicht eigentlich an die Masse, es sei denn, es handelt sich um Massenauflagen (z. B. bei Taschenbüchern usw.). Im Vergleich zu anderen Medien wie Presse, Rundfunk, Film usw. erreicht das Buch nur eine begrenzte Personenzahl. Verdient es deshalb schon eine gesondere Behandlung? Muß man sich angesichts einer solchen Formulierung nicht fragen, ob die Kirche die durch die Medien bewirkte kulturelle Wandlung überhaupt schon wahrgenommen hat? Es geht keineswegs darum, die Bedeutung des Buches als solches in Zweifel zu ziehen, nur weil es nicht die breite Masse erreicht. Als Vehikel von Wissen und Ideen und dank seines ästhetischen Eigenwertes übt das Buch nach wie vor einen tiefgreifenden gesellschaftlichen Einfluß aus, der durchaus das Interesse der Kirche an diesem Medium rechtfertigt. Da es sich indessen um ein spezifisches Interesse handelt, so scheint dieses sich sehr wohl von dem zu unterscheiden, das die Kirche den sozialen Kommunikationsmitteln im eigentlichen Sinne entgegenbringt ${ }^{4}$. Diese Differenzierung ist um so bedeutsamer, als alle im Titel IV zusammengefaßten Canones speziell Fragen der kirchlichen Kontrolle oder gar der Zensur betreffen, wie sie vor allem auf Bücher anwendbar sind.

Schon der erste Canon (822) läßt die Tendenz erkennen, die Medien ausschließlich in ihrer instrumentellen Funktion im Dienst der Kirche zu sehen, indem die 'Hirten' verpflichtet werden, bemüht zu sein, die sozialen Kommunikationsmittel bei der Erfüllung ihrer pastoralen Aufgabe 'anzuwenden' ( $\$ 1$ ). „Denselben Hirten obliegt die Sorge, die Gläubigen dahingehend zu belehren, daß sie zur Mitarbeit verpflichtet sind, damit der Gebrauch der sozialen Kommunikationsmittel von menschlichem und christlichem Geist belebt wird“ ( $(2)$. Schließlich werden die Gläubigen - insbesondere jene, die in irgendeiner Weise mit den Medien zu tun haben - dazu aufgefordert, Hilfen für das pastorale Handeln zu leisten, „damit die Kirche auch mit diesen Mitteln ihre Aufgabe wirksam ausübt" $(\$ 3)$. Die $\$ \S 2$ und 3 beziehen sich auf die Abschnitte 3 und 13 des Konzilstextes 'Inter mirifica', über die sozialen Kommunikationsmittel', wobei sie allerdings den instrumentellen Charakter der Medien besonders hervorheben. Die in $\$ 3$ geforderte pastorale Hilfeleistung ist im Zusammenhang zu den generellen pastoralen Pflichten der Gläubigen, wie sie in den Canones 211, 222 und 225 des Codex festgelegt sind, zu sehen. Mit dem Canon 823 bewegen 
wir uns schon mitten im Bereich der Zensur. Fast wörtlich übernimmt der Codex den Text des postkonziliaren Dekrets vom 19. März 1975 'Ecclesiae pastorum'6 über 'Die Aufsicht der Hirten über die Bücher'. Der Codex ergänzt den Text lediglich mit folgendem Hinweis auf den Gebrauch der sozialen Kommunikationsmittel: „Um die Unversehrtheit der Glaubenswahrheiten und der Sittenlehre zu bewahren, ist es Pflicht und Recht der Hirten der Kirche, darüber zu wachen, daß nicht durch Schriften oder den Gebrauch der sozialen Kommunikationsmittel Glaube oder Sitten der Gläubigen Schaden nehmen; ebenso können sie verlangen, daß von Gläubigen herauszugebende Schriften, die den Glauben oder die Sitten berühren, ihrem Urteil unterworfen werden; schließlich haben sie Schriften zurückzuweisen, die dem rechten Glauben oder den guten Sitten schaden" $(\$ 1)$.

Der zweite Paragraph dieses Canon präzisiert zudem, daß, wenn von den 'Hirten der Kirche' die Rede ist, sowohl die Bischöfe einzeln als auch deren Versammlung, etwa in Partikularkonzilien oder Bischofskonferenzen, gegenüber den Gläubigen ihrer Diözesen verpflichtet sind; geht es aber um die Gesamtheit des Volkes Gottes, so ist der Heilige Stuhl damit gemeint. Die Canones 824 bis 835 sowie 831 enthalten Einzelbestimmungen über die kirchliche Bücherzensur.

In dem im Auftrag der Canon Law Society of America herausgegebenen Codex-Kommentar fassen die Autoren die historischen Aspekte dieses Kapitels sehr treffend zusammen?. Sie rufen die einschlägigen Bestimmungen des CIC von 1917 über Bücherzensur in Erinnerung (cc. 1384-1405) und verweisen auf die Aufhebung des 'Index librorum prohibitorum' durch die beiden Dekrete der Kongregation für die Glaubenslehre vom $14.6 .1966^{8}$ und vom $19.3 .1975^{\circ}$; deren Inhalt ist mit einigen kleineren Veränderungen in den Codex von 1983 eingegangen.

Man verfügt somit über eine, auf eine lange Tradition gestützte, hinreichend detaillierte Regelung, die von der Sorge um die Bewahrung der Authentizität der Botschaft des Evangeliums getragen wird. Allerdings wäre es angebracht, die Errungenschaften dieser Tradition unter heutigen Gesichtspunkten auf ihre praktische Handhabbarkeit hin zu überprüfen, und dies inbesondere im Hinblick auf komplexere Vorgänge. Die jüngere Geschichte zeigt anhand der Probleme mit bestimmten Theologen (um nur etwa Namen wie H. Küng, E. Schillebeeckx oder C.E. Curran zu nennen), wie hochgradig delikat jene Verantwortung einzuschätzen ist, die den Ortsordinarien zukommt und mit welchen Schwierigkeiten gerechnet werden kann, wenn kompetente Gutachter (gemäß c. 830 \1) mit der Beurteilung von Büchern beauftragt werden sollen. Nichts desto weniger befinden wir uns hier jedoch im immerhin begrenzten Bereich der Printmedien und nicht bei den sozialen Kommunikationsmitteln generell.

In eben diesen begrenzten Bereich der Printmedien gehört auch das Verbot für Gläubige, „in Tageszeitungen, Zeitschriften oder anderen periodischen Veröffentlichungen, welche die katholische Religion oder die guten Sitten offenkundig anzugreifen pflegen", zu publizieren (c. 831 \1). Einzige Ausnahme: „es sei denn, es läge ein gerechter und vernünftiger Grund vor"; Kleriker 
jedoch und Ordensleute dürfen dies jedoch nur mit Erlaubnis des Ortsordinarius tun. Zudem dürfen Ordensleute nur mit Erlaubnis ihres höheren Ordensoberen Schriften publizieren, die sich mit Fragen der Religion und der Sitten befassen (c. 832). Die Mitwirkung von Klerikern und Ordensleuten „in Hörfunk und Fernsehen bei der Behandlung von Fragen... die die katholische Lehre oder die Sitten betreffen", wird durch Partikularnormen der Bischofskonferenzen (c. 831 \2) geregelt.

Das ist im wesentlichen der Inhalt des IV. Titels des III. Buches. Aber der Codex läßt es dabei nicht bewenden.

\subsection{Weitere Hinweise auf die Medien im Codex}

Dehnt man die entsprechenden Recherchen über Titel IV des III. Buches aus, so stößt man auf mehrere Canones, in denen die sozialen Kommunikationsmittel erwähnt werden. Meist handelt es sich um wichtige Präzisierungen, die durch frühere Lehrschreiben, vor allem durch Konzilsdokumente, inspiriert sind. Allerdings gibt es auch andere, deren praktische Relevanz kaum ersichtlich ist und die überflüssig erscheinen.

Erstmals werden die sozialen Kommunikationsmittel im Canon 666 erwähnt, und zwar im Zusammenhang mit den Rechten und Pflichten von Ordensleuten (IV. Kapitel des III. Teils von Buch II über das Volk Gottes): „Beim Gebrauch der sozialen Kommunikationsmittel soll die erforderliche Unterscheidung eingehalten und das gemieden werden, was der eigenen Berufung schädlich und für die Keuschheit der geweihten Person gefährlich ist“".

Baragli weist auf den aszetischen Charakter dieses Textes hin und bemerkt, daß man in früheren Dokumenten über die Priesterausbildung ähnliche Anweisungen finden konnte ${ }^{10}$. Doch selbst im Rahmen eines solchen Kontextes macht der negative Charakter des Canon 666 betroffen. Sollte der Gesetzgeber nicht viel mehr in Betracht ziehen, daß Ordensleute die Medien im Sinne einer Bereicherung ihrer eigenen Persönlichkeit nutzen können, anstatt sie vor möglichen Gefährdungen durch diese bewahren zu wollen? Ist es nicht gerade dieser positive Gesichtspunkt, der Fernsehgeräte und Radios, Zeitungen und Zeitschriften in den Freizeiträumen der Ordenshäuser rechtfertigt?

Etwas weiter im Codex, am Anfang des III. Buches, wo es um den Verkündigungsdienst der Kirche geht, stellt der Canon 747 hinsichtlich der Kirche fest, es sei „ihre Pflicht und ihr angeborenes Recht, auch unter Einsatz der ihr eigenen sozialen Kommunikationsmittel, unabhängig von jeder menschlichen Gewalt, allen Völkern das Evangelium zu verkündigen“. Hierzu drängen sich zwei Anmerkungen auf:

a) Die sozialen Kommunikationsmittel werden hier ausschließlich als Instrumente im Dienst des Evangeliums betrachtet;

b) Die Kirche beansprucht das Recht und die Pflicht, eigene Medien zu besitzen, also Tageszeitungen, Rundfunksender und Filmproduktionsstätten usw.

Selbst wenn man die Berechtigung dieses Anspruchs anerkennt -- was angesichts gewisser politischer Konstellationen, in denen der Staat sich das 
Medienmonopol angemaßt hat, durchaus plausibel und angemessen scheint -, so dürfte man doch einige differenziertere Grundpositionen im Hinblick auf die vielfältigen Möglichkeiten einer christlichen Präsenz im Bereich der sozialen Kommunikation erwarten. Man muß wissen, daß schon im Konzilsdekret 'Inter mirifica'(Artikel 3) derselbe Anspruch auf Nutzung und Besitz eigener Medien erhoben wird. Dieser Anspruch ist später in der Pastoralinstruktion 'Communio et Progressio' vom 21. Mai 1971 der Päpstlichen Kommission für die Instrumente der sozialen Kommunikation ${ }^{11}$ noch verdeutlicht worden.

Die Presse und die übrigen Medien sind gemäß c. 761 gleicherweise unter die Werkzeuge zur Verbreitung der christlichen Glaubenslehre einzureihen. Es handelt sich hier um einen Text, der nahezu wörtlich aus dem Konzilsdekret 'Christus dominus' über die Hirtenaufgabe der Bischöfe in der Kirche, Nr. 13 , entliehen ist ${ }^{12}$.

Im zweiten Kapitel des selben Titels, das der katechetischen Unterweisung gewidmet ist, finden wir den Canon 779, der zusammen mit c. 804 der einzige ist, der den Begriff der Instrumente (anstelle von 'Mittel') der sozialen Kommunikation ${ }^{13}$ gebraucht. Er empfiehlt die Verwendung dieser Instrumente neben den didaktischen Hilfen und Hilfsmitteln - für die katechetische Unterweisung.

Schon c. 772 fordert, daß hinsichtlich der Verbreitung der christlichen Lehre in Hörfunk oder Fernsehen die Vorschriften der Bischofskonferenz zu beachten sind ${ }^{14}$. Im Kapitel über die Schulen (Buch III, Titel II, Kapitel 1) findet sich c. 804, der den Religionsunterricht und die katholische Erziehung, die in den Schulen jeglicher Art vermittelt "Oder in den verschiedenen sozialen Kommunikationsmitteln ('instrumentis') geleistet werden" der kirchlichen Autorität unterstellt.

Baragli ist zuzustimmen, wenn er den Bezug auf diese „Instrumente" für überflüssig hält. „Beim derzeitigen Stand der Dinge“ schreibt er, „ist kaum zu sehen, wie sie zur katechetischen Unterweisung und einer systematischen religiösen Erziehung mehr als nur sehr allgemein und gelegentlich beitragen könnten $^{\prime 15}$. Trotz dieses Vorbehalts sollte man dennoch auf die vielfachen Bemühungen hinweisen, die beispielsweise in Lateinamerika darauf hinzielen, audiovisuelle Medien in den Dienst von systematischen und strukturierten religiösen Bildungsprogrammen zu stellen. Ein weiterer problematischer Hinweis auf die sozialen Kommunikationsmittel findet sich in c. 1063. Dort wird die Nutzung der Medien für die Ehevorbereitung empfohlen. Allerdings dürfte die Nutzung der Medien nicht zu Lasten des persönlichen Kontakts zwischen Seelsorgern und Gläubigen gehen.

Innerhalb des Buches VI über die Strafbestimmungen in der Kirche heißt es im Canon 1369, der Straftaten beim Gebrauch der Massenmedien anführt: „Wer in einer öffentlichen Aufführung oder Versammlung, entweder durch öffentliche schriftliche Verbreitung oder sonst unter Benutzung von sozialen Kommunikationsmitteln, eine Gotteslästerung zum Ausdruck bringt, die guten Sitten schwer verletzt, gegen die Religion oder die Kirche Beleidigungen aus- 
spricht oder Haß und Verachtung hervorruft, soll mit einer gerechten Strafe belegt werden“.

Auf diese Bestimmung, der dem c. 2323 des alten CIC von 1917 entstammt, hätten sich die Kritiker von M. Scorsese berufen können, als sie ihm kürzlich vorwarfen, mit „Die letzte Versuchung Jesu“ einen blasphemischen Film geschaffen zu haben. Allerdings hätten sie den Nachweis der Blasphemie liefern müssen. Und das dürfte einige Schwierigkeiten bereitet haben.

Nach Durchsicht von Titel IV des Buches III, der gänzlich den sozialen Kommunikationsmitteln (,insbesondere Bücher") gewidmet ist, haben wir auch bei etlichen anderen Canones Hinweise auf die Medien entdecken können. Der hieraus gewonnene Gesamteindruck ist dazu angetan, jene zu verunsichern, die von der täglich wachsenden Bedeutung der Medien für unsere Alltagskultur überzeugt sind. Ist doch im Kodex durchweg von den Gefahren, die von den Medien ausgehen, die Rede. Sie werden hier lediglich aus der Perspektive des instrumentellen Nutzens für diesen oder jenen pastoralen Dienst der Kirche behandelt. Jene durchaus möglichen Dienste, welche die Gesellschaft von der Kirche im Hinblick auf eine Belebung der kommunikativen Dimension ihrer kulturellen Entwicklung erwarten dürfte, werden jedoch praktisch mit Schweigen übergangen. Allerdings hat es durchaus positive Elemente in früheren Verlautbarungen des kirchlichen Lehramtes gegeben, von denen sich die Autoren des Codex bei der Abfassung dieser Texte hätten inspirieren lassen können.

\section{Was der Codex verschweigt}

Auch in diesem Punkt trägt die Arbeit von Baragli wichtige Details bei ${ }^{16}$. Zur Verdeutlichung teilen wir die Auslassungen des Codex in drei Gruppen, nämlich in jene, die sich auf kirchliche Strukturen, dann auf konkrete Aktivitäten und schließlich auf jene Probleme beziehen, die sich durch die Medien ergeben.

\subsection{Auslassungen binsichtlich kirchlicher Strukturen}

Sehr häufig verweist der Codex auf die kirchliche Autorität, und dies vor allem auf die päpstliche oder bischöfliche. Nirgendwo jedoch werden die kirchlichen Dienststellen der sozialen Kommunikation erwähnt, die doch auf Pius XI. zurückgehen, der in Nr. 48 seiner Enzyklika 'Vigilanti cura' vom 29. Juni $1936^{17}$ empfiehlt, daß die Bischöfe in jedem Land eine Filmzentrale schaffen sollten, um die Kinofilme nach ihrem sittlichen Wert zu beurteilen. Dementsprechend haben zahlreiche Länder nach recht verschiedenartigen Kriterien eine solche Einrichtung geschaffen. Die Einsetzung der Päpstlichen Kommission für die Instrumente der sozialen Kommunikation hat die entsprechenden Aktivitäten beschleunigt und hat zu einer Präzisierung der diesbezüglichen Modalitäten beigetragen.

Das Konzilsdekret 'Inter mirifica' vom 4. Dezember 1963 bestätigt Existenz und Kompetenz der Päpstlichen Kommission in Art. 21; die Pastoralinstruktion 'Communio et Progressia' vom 21. Mai 1971 präzisiert dies ihrerseits 
(in den Abschnitten Nr. 4, 168, 170-171 und 177). Demgemäß sollten wir über Texte von hinreichender Eindeutigkeit und Autorität verfügen können. Wir sollten! Die Praxis lehrt uns leider, daß diese Texte weitgehend unbeachtet geblieben oder in Vergessenheit geraten sind. Ihre Aufnahme - zumindest deren wesentliche Aussagen - in den Codex des kanonischen Rechtes wäre daher sehr wichtig gewesen. Man hätte damit Gelegenheit gehabt, die spezifischen Medien- und Kommunikationsstrukturen zu erfahren und näher zu beschreiben. Denn häufig werden die Medien von seiten der kirchlichen Stellen mehr als Hilfsmittel für ihre eigene Öffentlichkeitsarbeit instrumentalisiert, denn als eigengesetzliche Organismen betrachtet, die christliche Wertvorstellungen im Medienbereich präsent machen können. Es ist bedauerlich, daß der Codex gemäß seiner Funktion keine Aussage über die Aufgabenstellung des Päpstlichen Rates für die Instrumente der sozialen Kommunikation machen kann. Dieses Gremium wurde am 20. Januar 1948 von Pius XII. als „Päpstliche Kommission für den Unterrichtsfilm und den religiösen Film“18 gegründet; am 1. Januar 1952 wurde sie in „Päpstliche Filmkommission" ${ }^{19}$ umbenannt; am 16. Dezember 1954 wurde ihre Kompetenz auch auf die Gebiete von „Rundfunk und Fernsehen" ausgeweitet ${ }^{20}$. Sie wurde von Papst Johannes XXIII. durch das Motu proprio „Boni pastoris" vom 22. Februar 1959¹ bestätigt. Am 2. April 1964 gab Paul VI. der Kommission in seinem Motu proprio „In fructibus multis“22 ihre gegenwärtige Form, und schließlich wurde im Rahmen der Kurienreform vom 1. März $1989^{23}$ aus der „Kommission“ ein „Päpstlicher Rat“. Es handelt sich um ein Gremium mit höchster Kompetenz innerhalb der kirchlichen Struktur, dessen Erwähnung im Codex auch die Bedeutung seiner Aktivitäten dokumentiert hätte.

Ebenfalls zu bedauern ist, daß der Codex seiner Anlage gemäß auch die internationalen Fachverbände unerwähnt lassen mußte: OCIC, UNDA und $\mathrm{UCIP}^{24}$. Andererseits hat das Konzil sehr wohl den Päpstlichen Rat und die internationalen Medienverbände erwähnt; etwa in 'Inter mirifica' (Art. 19 und 22) und dann in 'Communio et Progressio' (Nr. 172 und 178-180). Zwar sind die internationalen Fachverbände hinreichend legitimiert durch c. 215 (wonach es den Gläubigen unbenommen ist, „Vereinigungen für Zwecke der Caritas und der Frömmigkeit und zur Förderung der christlichen Berufung in der Welt frei zu gründen und zu leiten“) und durch c. 216 (wonach die Gläubigen das Recht haben, „auch durch eigene Unternehmungen je nach ihrem Stand und ihre Stellung eine apostolische Tätigkeit in Gang zu setzen oder zu unterhalten"), aber dennoch hätten sie einen Platz im Codex verdient, um so mit größerer Autorität auftreten und handeln zu können.

\subsection{Aktivitäten}

Eine jener Aktivitäten, die seit mehr als zwanzig Jahren das Interesse der Kirche an den Medien markieren, ist der „Welttag der Kommunikationsmittel“, der durch 'Inter mirifica', Art. $18^{25}$ institutionalisiert worden ist. Er gibt dem Papst und den nationalen Bischofskonferenzen Gelegenheit zu Botschaften über das jeweils jährlich festgelegte Welttagsthema. Schon diese gesammelten Botschaften allein stellen einen wichtigen Aussagekorpus des kirchlichen Lehramtes dar. Dort treten auch zunehmend die positiven Gesichtspunkte zutage. Hinweise 
innerhalb des Codex auf diese und ähnliche andere kirchliche Medienaktivitäten hätten deren Breitenwirkung verstärken können (das gilt $z$. B. auch für den Weltfriedenstag, den Welttag der geistlichen Berufe und den Weltmissionssonntag usw.).

Ein anderes Medienphänomen, das in vielen Teilen der Welt aus verschiedenen Gründen an Bedeutung gewinnt, ist die Übertragung der Sonntagsmesse in Hörfunk und Fernsehen. Der Codex hätte diese Gottesdienstübertragungen erwähnen können, als er in c. $1248 \$ 2$ Empfehlungen ausspricht für den Fall, „wenn wegen Fehlens eines geistlichen Amtsträgers oder aus einem anderen schwerwiegenden Grund die Teilnahme an einer Eucharistiefeier unmöglich ist".

Hierzu schreibt Baragli: "Wenn auch der Kanontext nicht ausschließt, daß das "Gebet in der Familie oder ggf. in Familienkreisen“ an die Stelle der Teilnahme am Sonntagsgottesdienst treten könnte, so würde ein ausdrücklicher Hinweis auf Gottesdienstübertragungen in Hörfunk und Fernsehen doch eine Ermutigung darstellen, zumal sowohl das kirchliche Lehramt als auch das Konzil sich wiederholt positiv hierzu geäußert haben ${ }^{26}$. In einer Fußnote zählt er noch einige Beispiele hierfür auf. Die Teilnahme an einer Fernsehübertragung der Eucharistiefeier wirft Probleme auf, die über den rechtlichen Rahmen hinausgehen. Dennoch wird sie immer mehr Teil der gelebten Wirklichkeit vieler Gläubigen und verdient daher einen Platz im Codex des Kanonischen Rechtes ${ }^{27}$.

\subsection{Probleme}

Das schwerwiegendste Problem, das der Codex unterschätzt hat, ist wohl das des Rechts auf Information. Dies ist umso erstaunlicher, als gerade die Kirche es gewesen ist, die, wie J. Cousineau in seinem Kommentar zu 'Inter mirifica ${ }^{128}$ sagt, ,als erste internationale Körperschaft kompetent zu einem der vitalsten und brennensten Probleme unserer heutigen Gesellschaft Stellung genommen hat". Das Konzilsdekret sagt an dieser Stelle: „Es gibt also in der menschlichen Gesellschaft ein Recht auf Information über alle Tatsachen, die den Menschen als einzelnen oder als Mitglieder der Gesellschaft, je nach ihrer besonderen Situation zu wissen zukommt". Die Pastoralinstruktion 'Communio et Progressio' entwickelt diese Aussage weiter und spricht sogar von einer Pflicht, von sich aus Information zu suchen ${ }^{29}$.

Die allgemeine Problematik des Rechts auf Information stellt sich in spezieller Art und Weise hinsichtlich der Information innerhalb der Kirche. 'Inter mirirfica' äußert sich hierzu nicht. Doch 'Communicatio et Progressio' sagt hierzu in Nr. 119, daß ,jeder Gläubige das Recht und die Möglichkeit haben (muß), sich über alles zu informieren, was erforderlich ist, um im Leben der Kirche eine aktive Rolle zu übernehmen". Bedauerlicherweise geht der Codex nicht näher auf dieses Thema ein. Gewiß gibt es die Aussage des c. $212 \S 3$, wonach den Gläubigen „das Recht und bisweilen sogar die Pflicht" zusteht, ,ihre Meinung in dem, was das Wohl der Kirche angeht, den geistlichen Hirten mitzuteilen ...und ...den übrigen Gläubigen kundzutun". Aber zugleich wird diese Maßgabe auch wieder eingeschränkt, indem es heißt, dies habe „unter Wahrung der Unver- 
sehrtheit des Glaubens und der Sitten und der Ehrfurcht gegenüber den Hirten und unter Beachtung des allgemeinen Nutzens und der Würde der Personen“ zu geschehen.

Laien haben, nach c. 227 , "das Recht, daß ihnen in den Angelegenheiten des irdischen Gemeinwesens" (warum nicht auch innerhalb der Kirche?)" jene Freiheit zuerkannt wird, die allen Bürgern zukommt; beim Gebrauch dieser Freiheit haben sie dafür zu sorgen, daß ihre Tätigkeiten" (warum allein ihre und nicht auch die des Klerus?) „vom Geist des Evangeliums erfültt sind, und sich nach der vom Lehramt der Kirche vorgelegten Lehre zu richten" haben. Der Abschluß dieses Kanons gipfelt in der bedeutsamen Feststellung, die jedoch erstaunlicherweise nur für Laien zu gelten scheint: „....dabei haben sie sich jedoch davor zu hüten, in Fragen, die der freien Meinungsbildung unterliegen, ihre eigene Ansicht als Lehre der Kirche auszugeben“. Man kann nicht umhin sich vorzustellen, daß gewisse kirchliche Amtsträger, ja selbst solche von sehr hohem Rang, sehr wohl daran täten, diese Maßgabe zu respektieren.

Ein weiteres Problem, zu dem der Codex keine hinreichenden Lösungsvorschläge bereithält, betrifft die Medienausbildung. Sie wurde schon vom II. Vaticanum in 'Inter mirifica' empfohlen (Art. 15 bezieht sich auf die Medienschaffenden, Art. 16 auf die Mediennutzer). 'Communio et Progressio' enthält hierzu detailliertere Richtlinien ${ }^{30}$.

Was die Medienbildung für Priesteramtskandidaten angeht, so enthalten die Texte der Abschnitte 68 und 69 der Ratio fundamentalis institutionis sacerdotalis der Kongregation für das katholische Bildungswesen entsprechende Richtlinien. Sie sind in den von dieser Kongregation am 19. März $1986^{31}$ herausgegebenen Leitlinien für die Ausbildung der Priesteramtskandidaten hinsichtlich des Umgangs mit den Medien weiterentwickelt worden. Trotz all dieser Empfehlungen zögern noch viele der für die Priesterausbildung Verantwortlichen, sich aktiv für eine Ausbildung im Medienbereich einzusetzen. Entsprechende Empfehlungen für den Ausbildungsbereich wären innerhalb des Codextextes sicherlich von großer Wirkung.

Darüber hinaus finden sich im Codex keinerlei Vorgaben hinsichtlich einer grundsätzlichen und finanziellen kirchlichen Mitwirkung im Bereich der Massenmedien, ob es sich nun um eigentlich kirchliche Beteiligungen oder um Aktivitäten katholischer Laien in Zusammenarbeit mit Nichtkatholiken handelt, ob es sich um Kapitalbeteiligungen, die immer mehr an Bedeutung gewinnen, dreht, oder ob es darum geht, beispielsweise Satellitenkapazitäten zu schaffen. Die hieraus entstehende Problemlage kann sicherlich nicht allein mit Hilfe allgemeiner Richtlinien des Kanonischen Rechts gemeistert werden. Hierzu bedarf es ganz spezifischer Regelungen. Da stehen gewichtige Interessen auf dem Spiel; dabei handelt es sich nicht einmal allein um reine Finanzprobleme, sondern auch um Fragen der wirksamen Plazierung der Investitionen. Soll man beispielsweise den Schwerpunkt auf die Investitionen im technisch-materiellen Bereich legen oder soll man vielmehr im Bereich der Produktion von Programmen investieren, an denen die Medienbesitzer interessiert sind? Sicherlich ist es nicht Sache der Verfasser eines Kirchenrechts-Codex, diesbezüglich Entschei- 
dungen zu treffen. Aber sie hätten wenigstens einige Grundpositionen festlegen können. Auch das ist ein weiterer Posten auf der Wunschliste, die man gerne jenen unterbreiten möchte, die vielleicht einmal eine Revision des Codex zu bewerkstelligen haben.

\section{Zusammenfassung}

Unbeschadet aller Kritik kommt dem Konzilsdekret über die sozialen Kommunikationsmittel 'Inter mirifica' das Verdienst zu, das Phänomen der Massenmedien in die höchsten lehramtlichen Aussagen des Konzils integriert zu haben. Ausgehend von vorstehenden Untersuchungen kommen wir zu folgenden Schlußfolgerungen:

- Die entsprechenden Bestimmungen des Codex des kanonischen Rechtes anerkennen - auf indirekte Art und Weise - die Problematik der zwischenmenschlichen Kommunikation als ein unverzichtbares Element innerhalb der kirchlichen Rechtsmaterie. Das ist erfreulich, denn eine derartige Anerkennung ist für alle diejenigen von Bedeutung, die in diesem Bereich tätig sind.

- Eine grobe Überprüfung der Kodextexte veranlaßt jedoch zu der Festellung, daß sie leider nicht jene Grundaussagen reflektieren, die in früheren lehramtlichen Dokumenten getroffen sind. Die päpstlichen Dokumente 'Inter mirifi$\mathrm{ca}^{\prime}$, die Pastoralinstruktion 'Communio et progressio' enthalten eine ganze Reihe von Richtlinien, die gut und gerne in den Codex aufgenommen gehörten und die im Kontext zu den anderen Themenbereichen einen prominenten Platz eingenommen hätten.

- Selbst wenn man die früheren Aussagen auch als rechtlich relevant betrachtet, so muß doch festgestellt werden, daß ihre Nichterwähnung im Codex ihrer praktischen Anwendung keinesfalls förderlich ist.

- Was davon im Codex allerdings weit verstreut auffindbar ist, widerspiegelt zweierlei Vorurteile: a) die der möglichen Gefährdungen, die die Medien darstellen und b) die der Möglichkeiten, die Medien rein instrumentell in den Dienst der Evangelisierung zu stellen. Beide Gesichtspunkte sind durchaus legitim. Aber sie entsprechen einer durchweg negativen und defensiven Sicht des neuen Phänomens der Medien, eines Phänomens, das bereits heute unser individuelles und gesellschaftliches Leben dominiert.

- Man kann der Kirche durchaus zugestehen, daß sie die Gefahren wahrnimmt, die von den Medien ausgehen, und daß sie die Möglichkeiten nutzt, die ihr damit für die Verkündigung ihrer Botschaft gegeben sind. Aber sie darf nicht, als Mater et Magistra, als Mutter und Lehrmeisterin, die sie ist, aus dem Blickfeld verlieren, wie sehr sie den Bedürfnissen jener Menschen verpflichtet ist, die ihr Leben im Umgang mit den Medien zu bereichern und zu erfüllen trachten. Die Kirche kann den Menschen dienen, indem sie ihnen zeigt, wie sie die durch die Medien gegebenen Möglichkeiten für ihre menschliche und für ihre persönliche Entwicklung nützen können. Dadurch 
würde die Kirche an Vertrauen und Glaubwürdigkeit gewinnen, was ihrer eigentlichen Heilsbotschaft zugute käme.

- Gegen Schluß der römischen Bischofssynode über das Laienapostolat (1987) wurden etliche Stimmen laut, die forderten, die nächste Bischofssynode solle gänzlich dem Thema der sozialen Kommunikationsmittel gewidmet sein. Wir haben gegenüber diesem Vorschlag einige Vorbehalte. Denn für viele Bischöfe ist diese Thematik noch zu wenig vertraut. Bevor man sich auf einer Synode auf ein solches Thema einläßt und dabei riskiert, mit einigen eilfertigen Beschlüssen davonzukommen, die doch wieder Haltungen festschreiben, die längst passé sein sollten, sollte man beharrlich weitererarbeiten und zwar mit dem Ziel, ein neues päpstliches Dokument zu erstellen, das sich auf die Erfahrungen der vergangenen Jahre seit 'Inter mirifica' und 'Communio et progressio' gründet.

- Gerade in letzterem Dokument liegt uns ein Text vor, der eine Fülle von Möglichkeiten für einen neuen Zugang zu den Medien bietet. Es wäre an der Zeit, die notwendigen Folgerungen daraus zu ziehen, damit sich eine veränderte Haltung gegenüber den Medien ausbreitet und daß der Boden bereitet wird für ein fruchtbares Gespräch auf einer nächsten Bischofssynode.

- Bis es soweit sein wird, müssen wir alles tun, damit die einschlägigen Bestimmungen des Codex des kanonischen Rechtes mit großer Sensibilität gegenüber der sozialen Kommunikation verstanden und angewendet werden, wie es auch der universellen Lehre der Kirche entspricht, wenn es um Auftrag und Sendung zur Förderung des Menschen im Licht des Evangeliums geht.

\section{Anmerkungen:}

$1 \mathrm{Zu}$ den einschlägigen Bestimmungen im CIC von 1917 vgl. M. Schmolke, Die schlechte Presse, Münster/W. 1971, S. 206.

2 A. Ruszkowski. „Décret sur les communications sociales: succès ou échec du Concile?" in R. Latourelle (Hrsg.). Vatican II: bilan et perspective vingt-cing ans après (1962-1987), Band 3, Montreal. Ed. Bellarmin, Paris, Ed. du Cerf. 1988, S. 535-562.

3 „I 'Mass media'nel nuovo Coice di diritto canonico“ in La Civiltá cattolica , Jg. 136, Bd. 3 (Juli-September 1983), S. 209-222. Vom gleichen Autor siehe auch: Cinema cattolico: documenti della S. Sede sul cinema (Collana Magisterium. I). 2. Ausg. Roma Città nuova Editrice. 1965. 437 S.. Comunicazione, comunione e Chiesa (Collana Magisterium, 4) Roma. Studio romano della comunicazione sociale. 973. 1447 S. Difendo inter mirifica, Catania. Ed. Paoline, 1974, 119 Ss. L'Inter mirifica: introduzione, storia, discussione, commento, documentazione (Collana Magisterium, 2) Roma. Studio romano delle comunicazione sociale. 1969. $676 \mathrm{~S}$.

4 Vgl. Baragli, „I 'Mass media'nel nuovo Codice di diritto canonico". S. 218.

5 Die katholische Kirche ist von Christus dem Herm gegründet, um allen Menschen das Heil zu bringen, und ist darum der Verkündigung des Evangeliums unbedingt verpflichtet. Deshalb hält sie es für einen Wesensteil ihrer Aufgabe, die Heilsbotschaft auch mit Hilfe der publizistischen Mittel zu verkündigen, sowie Grundsätze über deren richtige Anwendung aufzustellen.

Der Kirche kommt also das ursprüngliche Recht zu, jede Art dieser publizistischen Mittel, sofern diese für die christliche Erziehung und jede ihrer Bemühungen zum Heile der Seelen notwendig oder nützlich sind, zu benutzen und zu besitzen. Zum Auftrag der Oberhirten gehört es, die Gläubigen zu lehren und zu leiten, damit sie, auch durch die Hilfe dieser Mittel, für sich und die ganze Menschheitsfamilie das Heil 
und die Vollendung erlangen.

Im übrigen ist es vor allem die Aufgabe der Laien, die publizistischen Mittel mit dem Geist der Menschlichkeit und des Christentums zu beseelen, um so den großen Erwartungen der Menschheit und dem Plane Gottes zu entsprechen." (Nr. 3 in AAS. 56, 1964, S. 146). „Alle Glieder der Kirche sollen einmütig und planmäßig, ohne Aufschub und mit größtem Eifer die publizistischen Mittel in den vielfältigen Arbeiten des Apostolats orts- und zeitgerecht benutzen und schädlichen Unternehmungen zuvorkommen. Das soll vor allem in den Gegenden geschehen, deren sittlicher oder religiöser Fortschritt erhöhte Anstrengungen erfordert.

Die Oberhirten sollen auch auf diesem Gebiet, das mit ihrer Pflicht zur Verkündigung so eng verbunden ist, ihren Auftrag erfüllen. Auch die mit den publizistischen Mitteln befaßten Laien sollen für Christus Zeugnis ablegen. Das geschieht vor allem durch Erfüllung ihrer jeweiligen Berufsaufgaben mit Sachverstand und apostolischem Geiste, ferner auch, je nach Möglichkeit, durch unmittelbare Unterstützung der kirchlichen Seelsorge mit ihren technischen, wirtschaftlichen, kulturellen und künstlerischen Kräften." (Nr. 13 in AAS. 56/1964, S. 149-150).

6 Einführung Abschnitt 2, in AAS. 67 (1975) S. 281.

7 J.A. Coriden. ,The Teaching Office of the Church (cc. 747-833)" in J.A. Coriden, T.J. Green, D.E. Heintschel (Hrsg.), The Code of Canon Law. A Text and Commentary, commissioned by the Canon Law Society of America, New York, Mahwah, NJ. Paulist Press, 1985, S. 578-585. Vgl. auch H. Mussinghoff, „Die sozialen Kommunikationsmittel. Besonders Bücher" in K. Lüdicke (Hrsg.), Münsterischer Kommentar zum Codex iuris canonici, Essen, Ludgerus Verlag, 1985 zu cc. 822-832.

8 Dekret "Post litteras apostolicas“ in AAS. 58 (1966), S. 445.

9 Dekret "Ecclesiae pastorum" in AAS. 67 (1975), S. 281-284.

10 „I 'Mass media' nel nuovo Codice di diritto canonico", S. 215.

11 AAS. 63 (1971), S. 593-656. Vgl. Art. 132: „Um ihre Absichten und Vorstellungen durch die Medien öffentlich zu machen, bieten sich der Kirche zwei Wege: entweder sie erhält aufgrund bestimmter Vereinbarungen Zugang zu Kommunikationsmitteln, die sie nicht selbst besitzt, oder sie bedient sich je nach Lage der Dinge eigener Kommunikationsmittel. Dabei kann man nicht absehen von Voraussetzungen, die je nach Land und Medium unterschiedlich sind. Die kirchlichen Vorgesetzten müssen ihren Beauftragten sachgerechte Weisungen geben, nachdem sie sich zuvor mit Sachverständigen auf örtlicher, nationaler und ggf. internationaler Ebene eingehend beraten haben." Vgl. auch Nr. 126.

12 Vgl. Baragli „I 'Mass media' nel nuovo Codice di diritto canonico“, S. 216.

13 Eine Diskussion um die Vorzüge des einen oder des anderen Begriffs scheint uns hier überflüssig zu sein.

14 Anmerkung der Redaktion: Die Deutsche Bischofskonferenz hat 1984 entsprechende Partikularnormen zu c. $772 \$ 2$ (wie auch c. $831 \$ 2$ ) erlassen. Damit wird die kirchliche Verantwortungsstruktur - von den zuständigen Diözesanbischöfen zu den jeweils zuständigen kirchlichen Senderbeauftragten - geregelt. Diese Regelungen betreffen auch die Qualifikationen und die kirchliche Legitimation von bei der Lehrverkündigung über den Rundfunk mitwirkenden Personen sowie die Übertragungen von Gottesdienstfeiern und liturgischen Handlungen.

15 "I 'Mass media' nel nuovo Codice di diritto canonico“ S. 218.

16 Ebd. S. 220-222.

17 AAS. 28 (1936) S. 261.

18 Das Gründungsdokument der Kommission ist nie offiziell veröffentlicht worden. Baragli macht (in "Cinema cattolico" doc. 39, S. 119) eine entsprechende Andeutung.

19 Das Dokument über die Namensänderung und die Aufgabenstellung der Kommission ist nicht veröffentlicht worden.

20 AAS. $46(1984)$, S. 783-784.

21 Ebd. 51 (1959), S. 183-187.

22 Ebd. 56 (1964), S. 289-292.

23 Apostolische Konstitution „Pastor bonus“ vom 29. Juni 1988, Nr. 169-170 in ebd. 80 
(1988), S. 904-905.

24 OCIC = Internationale katholische Organisation für Film und audiovisuelle Medien. UNDA (das lateinische Wort für "Welle") = Internationale katholische Vereinigung für Hörfunk und Fernsehen. UCIP = Katholische Weltunion der Presse.

25 „Um das vielgestaltige Apostolatswerk der Kirche auf dem Gebiet der Publizistischen Mittel wirksam zu kräftigen, soll in allen Diözesen des Erdkreises nach dem Ermessen der Bischöfe jährlich ein Tag festgesetzt werden, dieser Fragen im Gebet zu gedenken und Spenden für sie zu entrichten. Der Erlös soll zum Unterhalt und zur Unterstützung aller kirchlichen Einrichtungen auf diesem Gebiet dienen. Maßgebend sind dabei die Bedürfnisse der Weltkirche." (AAS. 56/1964, S. 151). Vgl. auch Communio et progressio, Nr. 100, in AAS. 63 (1971), S. 629.

26 "I 'Mass media' nel nuovo Codice di diritto canonico“, S. 222.

27 Anmerkung der Redaktion: Die Deutsche Bischofskonferenz hat in ihren Partikularnormen (vgl. Fußnote 12a) diesem Anliegen insoweit Rechnung getragen, als sie in Nr. 4 ihrer Partikularnormen zu c. $772 \$ 2$ festlegt: „Meßsfeiern dürfen nur live und nur vollständig übertragen werden; sie sind kein Ersatz für solche Meßfeiern, die von den Gläubigen in räumlicher Gegenwart mitzufeiern sind."

28 Vgl. J. Cousineau „Eglise et mass média: l'évolution des attitudes sur les communications sociales chez les hautes autoritês de l'Eglise catholique“ (Cahiers d'études et de recherches Nr. 16), Montreal, Office des communications sociales, 1973, $45 \mathrm{f}$.

29 „Dem Recht, das aus diesen Erfordernissen erwächst, entspricht auf der anderen Seite die Pflicht, von sich aus Information zu suchen. Denn das Informationsrecht bliebe nur Formel, wenn sich nicht jeder bemüht, auch informiert zu sein." (Nr. 34, in AAS. 63/1971, S. 606).

30 Vgl. Ruszkowski, „Décret sur les communications sociales", S. 556-557.

31 Ebd. Die Pastoralinstruktion enthält unter anderem genauere Richtlinien in den Nr. 15, 101-102 und 107 (AAS, 63/1971, S. 559-600; 629-630 und 631-632).

\section{SUMMARY}

The article presents an analysis of the statements on the means of social communication which are made in the Code of Canon Low revised in 1983. Several basic tenets contained in documents of the Magisterium (as for example in "Inter mirifica“ and "Communio et progressio") have not been included. The regulations made by the canon law convey a consistently negative and defensive impression of the phenomenon "media". In comparison with the afore-mentioned documents, the Code of Canon Law is far more reticent about the right to information. The guidelines which refer to the training of future priests in the media and which are contained in ${ }_{n}$ Communio et progressio" have not been included into the Code of Canon Law. According to the authors of the article, this formulation of the canons may also be a reason for the conflicts between the magisterium and professors of theology. The distinction which should be made between clergymen and laity, with reference to the possible use of and committment in the media, is considered to be unclear in the Code.

\section{RÉSUMÉ}

L'article présente une analse des déclarations relatives aux moyens de communication sociale contenues dans le Code de droit canoique révisé en 1983. Plusieurs déclarations fondamentales faites dans des documents du Magistère (comme par exemple dans „Inter mirifica“ et "Communio et progressio“) n'ont pas été prises dans le nouveau Code. Les règles contenus dans le droit canonique donnent une impression généralement négative et défensive du phénomène des médias. Comparé aux documents mentionnés ci-dessus, le Code est beaucoup plus éticent concernant le droit à l'information. Les directives que le document "Communio et progressio“ donne au sujet de la formation 
des futurs prètres dans le domaine des médias n'ont pas été pris dans le Code. D'après 1 'opinion des auteurs de cet article, cette mise au point des canons peut aussi ètre une raison pour les conflits entre le Magistère et des professeurs de théologie. Ils considèrent qu'il y a un manque de distinction qui devrait ètre faite entre le clergé et les laics concernant l'usage des médias et l'engagement dans ce secteur.

\section{RESUMEN}

El artículo presenta un análisis de las declaraciones acerca de los medio sde comunicación social contenidas en el Código del derecho canónico revisado en 1983 . La nueva versión no incluye una serie de declaraciones fundamentales comprendidas en documentos del Magisterio (tal como en "Inter mirifica“ y "Communio et progressio"). Las reglas contenidas en el derecho canónico reflejan una imagen generalmente negativa y defensiva del fenónemo de los medios. En comparación con los documentos ya mencionados, el Código es mucho más moderado en cuanto al derecho a información. El Código no incluye las directrices acerca de la formación de seminaristas en el campo de los medio sformuladas en el documento "Communio et progressio". Según los autores del artículo, la formulcación presente de los cánones puede dar también motivo a conflictos entre el Magisterio y profesores de teología. En su opinión, no es muy precisa la distinción entre los clérigos y los laicos en lo que se refiere al uso posible de los medios y al compromiso realizable en este campo. 